\title{
Impact of Conjunctival Folds on Central Tear Meniscus Height
}

\author{
Heiko Pult ${ }^{1-3}$ and Britta Helen Riede-Pult ${ }^{1,2}$ \\ ${ }^{1}$ Dr. Heiko Pult - Optometry and Vision Research, Weinheim, Germany \\ ${ }^{2}$ School of Optometry and Vision Sciences, Cardiff University, Cardiff, United Kingdom \\ ${ }^{3}$ Ophthalmic Research Group, Life and Health Sciences, Aston University, Birmingham, United Kingdom
}

Correspondence: Heiko Pult, Steingasse 15, 694699 Weinheim, Germany;

ovr@heiko-pult.de.

Submitted: October 21, 2014

Accepted: January 10, 2015

Citation: Pult H, Riede-Pult BH. Impact of conjunctival folds on central tear meniscus height. Invest Ophthalmol Vis Sci. 2015;56:1459-1466. DOI:10.1167/iovs.14-15908
Purpose. To analyze the effect of temporal and nasal lid-parallel conjunctival folds (LIPCOF) on the central tear meniscus height (TMH).

Methods. Tear meniscus height of 40 eyes of 20 subjects ( 15 female, median age: 39 years) was evaluated by the Tearscope Plus. Temporal and nasal LIPCOF scores were observed by slit lamp (SL) examination. The cross-sectional LIPCOF area (LA) was measured by a modified Scheimpflug camera. Tear meniscus was colored by fluorescein and TM fluorescence (TMF) was evaluated by SL using a 5-grade pictorial scale. Tear meniscus fluorescence was defined by its intensity, height, and regularity at the LIPCOF area in comparison to the central TMF. Correlations between variables were analyzed by Pearson correlation for parametric data or Spearman rank for nonparametric variables.

Results. Inferiorly, central TMH was significantly correlated to temporal LA (Pearson: $r=$ $0.519, P=0.001)$ and nasal LA $(r=0.567, P=0.002)$. Superiorly, central TMH was significantly correlated with temporal LA $(r=0.352, P=0.033)$, but not with nasal LA $(r=$ $0.355, P=0.075$ ). Temporal LA was significantly correlated with temporal TMF (Spearman rank: $r=0.789, P<0.001)$, and nasal LA was significantly correlated to nasal TMF ( $r=0.415$, $P=0.008$ ). Scores of LIPCOF were significantly correlated with TMF scores (Spearman rank: temporal, $r=0.837, P<0001$; nasal, $r=0.609, P<0.001)$. Subjective LIPCOF grading was significantly correlated with LA (Spearman rank: temporal, $r=0.707, P<0.001$; nasal, $r=$ $0.515, P=0.001)$.

Conclusions. Lid-parallel conjunctival folds impact TMH evaluation. In patients with LIPCOF, the real tear film volume may be smaller than the TMH measurement suggests.

Keywords: lid parallel conjunctival folds, tear meniscus, conjunctivochalasis, Scheimpflug camera
R ulbar conjunctival folds were probably first described by Middlemore in $1835 .{ }^{1}$ He used the term "conjunctival fold" to describe bulbar conjunctival folds visible with the naked eye. In 1942, Hughes ${ }^{2}$ named those severe bulbar conjunctival folds treatable by surgery as "conjunctivochalasis." However, use of the term "chalasis" implies a relationship between age and the conjunctival folds; and for conjunctival folds at the small, subclinical level, age does not appear to be correlated with their formation. ${ }^{3}$ Hence, Höh et al. ${ }^{3}$ described this latter type as lid-parallel conjunctival folds (LIPCOF). Lid-parallel conjunctival folds are folds in the lateral, lower quadrant of the bulbar conjunctiva, parallel to the lower lid margin. Lid-parallel conjunctival folds may represent the first mild stages of conjunctivochalasis and thus may share the same etiology. To avoid confusion, in this study LIPCOF refers only to subclinical conjunctival folds observed at a defined location, being unaltered after forced blinks, observed without fluorescein instillation, and classified by the optimized LIPCOF grading scale. ${ }^{4-7}$

Lid-parallel conjunctival folds are significantly related to dry eye. , $6,8-10$ Decreased mucin production is associated with the severity of LIPCOF, $^{5}$ and LIPCOF are significantly correlated with lid-wiper epitheliopathy. ${ }^{4-6}$ Lid-parallel conjunctival folds are treatable (for example, by using a liposomal eye spray) ${ }^{11}$ and experienced contact lens wearers with an increased LIPCOF score showed significantly lower LIPCOF degrees after 3 months, having been refitted with Senofilcon A contact lenses (Johnson \& Johnson Vision Care, Inc., Jacksonville, FL, USA). ${ }^{12}$

The evaluation of the tear meniscus appears to be an important diagnostic test, especially in the evaluation of the aqueous deficient dry eye. The tear menisci hold approximately $75 \%$ to $90 \%$ of the tear film volume, and the tear meniscus height is significantly correlated to tear film volume. ${ }^{13-18}$

Conjunctivochalasis can be located temporally, centrally, and nasally within the palpebral aperture, and therefore can present behind the full length of the inferior tear meniscus. Gumus et al. ${ }^{19}$ reported central tear film pooling at the lower tear meniscus in conjunctivochalasis. Wang et al. ${ }^{20}$ found an association between nasal conjunctivochalasis and delayed tear clearance. Lid-parallel conjunctival folds may also have an impact on the tear meniscus volume and the tear film drainage. Lid-parallel conjunctival folds occur behind the temporal and nasal tear meniscus along two-thirds of the total length of the inferior tear meniscus, and the paracentral tear menisci are affected by LIPCOF. $^{21}$ In fact, the paracentral tear meniscus is 
TABLE 1. Optimized Grading Scale of $\mathrm{LIPCOF}^{4-6,22}$

LIPCOF Grade

No conjunctival folds

1 permanent and clear parallel fold

2 permanent and clear parallel folds (normally lower than $0.2 \mathrm{~mm}$ )

More than 2 permanent and clear parallel folds (normally higher than $0.2 \mathrm{~mm}$ )

0

higher and flatter when increased LIPCOF scores are observed, compared with when they are absent. ${ }^{21}$

However, according to current knowledge, it is not yet clear if, and to what extent, LIPCOF impacts on central tear meniscus height measurements. This study aimed to analyze the effect of temporal and nasal LIPCOF on the central tear meniscus height.

\section{METHODS}

Tear meniscus height of 40 eyes of 20 subjects (15 female, median age: 39 years) was evaluated by the Tearscope Plus (Keeler Ltd., West Berkshire, UK). Temporal and nasal LIPCOF scores were observed by slit lamp examination. The crosssectional LIPCOF area was measured by use of a modified Scheimpflug camera (Pentacam HD; Oculus Optikgeräte GmbH, Wetzlar, Germany). Tear meniscus was colored by fluorescein, and tear meniscus fluorescence was evaluated by slit-lamp microscope using a 5-grade pictorial scale.

\section{Inclusion and Exclusion Criteria}

Subjects were excluded if they had abnormal lid margins, were younger than 18 years, had any history of ocular surgery, used any medication or eye drops known to affect the ocular surface, wore contact lenses, were pregnant, or if conjunctival folds increased after forced blinks. All procedures were conducted in accordance with the tenets of the Declaration of Helsinki (2000), and approval for the study was given by the Cardiff School of Optometry and Vision Sciences Ethics Committee. All subjects gave written informed consent before participating in the study.

\section{Lid-Parallel Conjunctival Folds}

Lid-parallel conjunctival folds were evaluated, without fluorescein, on the bulbar conjunctiva in the area perpendicular to the temporal and nasal limbus, above the lower lid (temporal and nasal LIPCOF, respectively), with a slit-lamp microscope using a $\times 25$ magnification.5,6,22 The patient's gaze was controlled by fixation points attached to the slit lamp microscope in order to guarantee straight-ahead fixation. Lid parallel conjunctival folds were classified using the optimized LIPCOF grading scale of Pult et al..$^{6,22}$ (Table 1). ${ }^{5}$ Care was taken to differentiate between parallel, permanent, conjunctival folds (LIPCOF) and disrupted microfolds (Markoulli M, et al. IOVS 2007;48:ARVO E-Abstract 5391 and Thota S, et al. IOVS 2006; 47:ARVO E-Abstract 82). ${ }^{5,6,22,23}$ This was done by evaluation of the fold thickness; the thickness of a single LIPCOF is approximately $0.08 \mathrm{~mm}$, while that of a microfold is $0.01 \mathrm{~mm}$ (Pult H and Riede-Pult BA, IOVS 2011;52:ARVO EAbstract 3739). The Scheimpflug technology was used in order to measure temporal and nasal LA at the previously described area of observation. The patient's gaze was controlled by fixation points attached to the Scheimpflug camera. The lid-

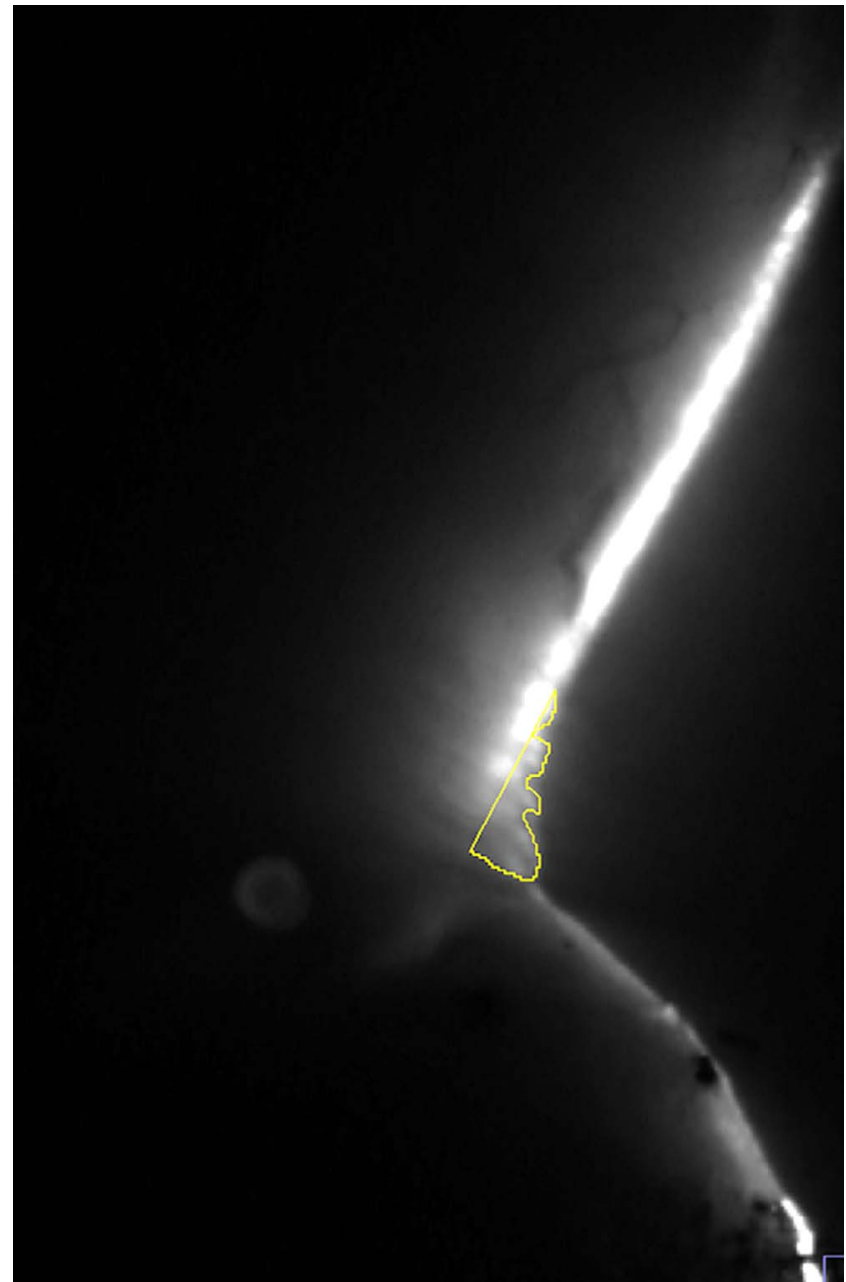

Figure 1. Evaluation of the cross-sectional area of LIPCOF.

parallel conjunctival folds were measured from the crosssectional area of the Pentacam scans (Fig. 1) using ImageJ software (http://imagej.nih.gov/ij/; provided in the public domain by the National Institutes of Health [NIH], Bethesda, MD, USA). This latter analysis was necessary because the LIPCOF images were distorted as a result of the camera angle and the automatic correction of the Scheimpflug images for the refractive indices of the ocular surface tissues. The Pentacam analysis tool would normally deal with this issue, but there was no tool available to measure the cross-sectional area of LIPCOF. To allow precise image analysis, the Oculus software was modified using ImageJ software (NIH). The software was additionally adapted by adding an option to amend the photographing exposure time and camera rotation, without which the folds would have been overexposed. The rotation was limited to $90^{\circ}$ to achieve consecutive images along the LIPCOF every $2^{\circ}$ (50 images). Lid-parallel conjunctival folds were always analyzed based on the image where the Scheimpflug camera slit was positioned perpendicularly to the lower lid margin. The slit lamp observation of LIPCOF was masked against the Pentacam analysis.

\section{Tear Film}

The tear film was observed 15 minutes after the evaluation of LIPCOF. The tear meniscus was illuminated by Tearscope, and images of both upper and lower tear menisci were taken by 


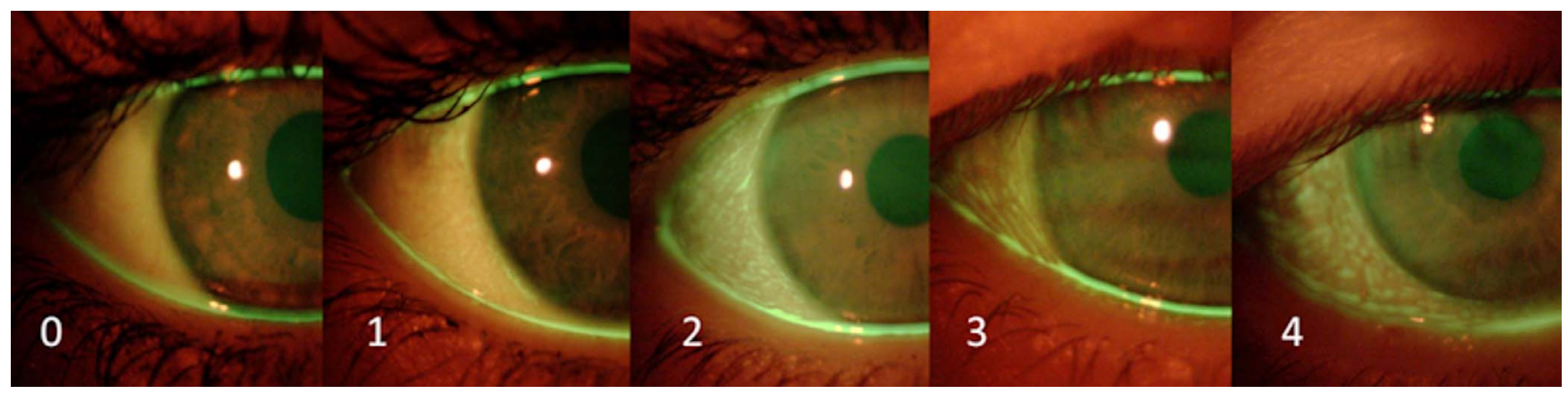

FIgURE 2. Tear meniscus fluorescence grading scale: Tear meniscus fluorescence was defined by TMF intensity, height, and regularity at the LIPCOF area in comparison with the central TMF (degree 0: regular TMF along the complete lower lid, degree 4: significant TMF interruption at LIPCOF area, compared with normal central TMF).

digital slit lamp microscope camera. The height of the central tear meniscus was measured using software (Phoenix; bon, Lübeck, Germany) developed for use with the slit lamp microscope camera (DigiPro HD; bon). Next, the tear film was colored using fluorescein and again photographed using the digital slit lamp microscope, enhanced with a cobalt blue illumination filter and a yellow (Wratten) barrier observation filter. Fluorescein was instilled applying the modified fluorescein strip procedure. ${ }^{24}$ This optimized application procedure was reported to significantly improve repeatability of fluorescein instillation when using fluorescein strips. ${ }^{24}$ The observer was instructed to prepare each fluorescein strip as follows:

1. Fold at $1 \mathrm{~mm}$ (perpendicularly to the longer side of the strip) from the end of the strip before removing it from its packaging;

2. Remove it from the packaging;

3. Moisten the fluorescein strip with a single drop of saline (Software Saline; Alcon, Großostheim, Germany);

4. Shake the strip, in order to remove excess fluorescein; and

5. Instill the fluorescein on the superior conjunctiva by slightly touching the conjunctiva with the folded part of the fluorescein strip.

Tear meniscus regularity was observed, based on the tear meniscus fluorescence. This test was named the tear meniscus fluorescence test. The tear meniscus fluorescence was defined by its intensity, height, and regularity at the LIPCOF area in comparison with the central tear meniscus fluorescence, using a pictorial grading scale (Fig. 2; degree 0: uninterrupted tear meniscus fluorescence along the complete lower lid; degree 4: significant tear meniscus fluorescence interruption at LIPCOF area, compared with normal central the tear meniscus fluorescence).

All images were stored and analyzed at a later time point in order to mask them to all other observations.

\section{Statistical Analyses}

Data were analyzed for normal distribution by KolmogorovSmirnov test and correlations between variables were analyzed by Pearson correlation for parametric data or Spearman rank for nonparametric variables (SPSS 20; SPSS, Inc., Chicago, IL, USA). The sample size was evaluated prior to the study by power calculation based on a power of $1-\beta=0.80(r=0.40, \alpha=$ $0.05)$. Since there are-to our best knowledge-no similar studies published, the expected effect $(r)$ of this pilot study was assumed from our knowledge of the field and previous investigation of the effect of LIPCOF on paracentral tear meniscus. $^{21}$

\section{Results}

Median central tear meniscus height was $0.30 \mathrm{~mm}$ at the lower lid and $0.20 \mathrm{~mm}$ at the upper lid; median temporal LIPCOF score was 1.5 and median nasal LIPCOF score was 0 . Median LIPCOF area was $34,984.3 \mu \mathrm{m}^{2}$ and $6289.3 \mu \mathrm{m}^{2}$ (Table 2), and median tear meniscus fluorescence score was 3 and 0 (temporally and nasally, respectively).

Significant correlations were observed between temporal and nasal LIPCOF scores, as well as temporal and nasal LIPCOF area (Spearman rank: LIPCOF, $r=0.454, P=0.003$; Pearson: LIPCOF area, $r=0.712, P<0.001$ ), but no correlation was found between the inferior, central tear meniscus height, and the superior tear meniscus height (Pearson: $r=-0.027, P=$ 0.471).

Inferiorly, central tear meniscus height was significantly correlated to temporal LIPCOF area (Pearson: $r=0.519, P=$ $0.001)$ and nasal LA $(r=0.567, P=0.002$; Figs. 3, 4). Superiorly, central tear meniscus height was significantly correlated with temporal LIPCOF area $(r=0.352, P=0.033)$, but not with nasal LIPCOF area $(r=0.355, P=0.075)$.

Temporal LIPCOF area was significantly correlated with temporal tear meniscus fluorescence (Spearman rank: $r=$

TABLE 2. Median and Quartiles of LIPCOF Area Ordered by Subjective LIPCOF Grades

\begin{tabular}{|c|c|c|c|c|c|c|c|}
\hline \multirow[b]{2}{*}{ Temporal LIPCOF } & \multicolumn{3}{|c|}{ Temporal LA, $\mu \mathrm{m}^{2}$} & \multirow[b]{2}{*}{ Nasal LIPCOF } & \multicolumn{3}{|c|}{ Nasal LA, $\mu \mathbf{m}^{2}$} \\
\hline & $25 \%$ & Median & $75 \%$ & & $25 \%$ & Median & $75 \%$ \\
\hline 0 & 0.00 & 0.00 & $13,757.86$ & 0 & 0.00 & $4,192.87$ & $11,923.48$ \\
\hline 1 & $10,220.13$ & $18,867.92$ & $36,949.69$ & 1 & 196.54 & $16,378.41$ & $45,007.86$ \\
\hline 2 & $43,435.53$ & $52,935.01$ & $75,340.67$ & 2 & $20,440.25$ & $57,914.05$ & $117,138.36$ \\
\hline 3 & $38,784.07$ & $77,175.05$ & $138,037.21$ & 3 & $31,970.65$ & $195,099.58$ & $358,228.51$ \\
\hline
\end{tabular}




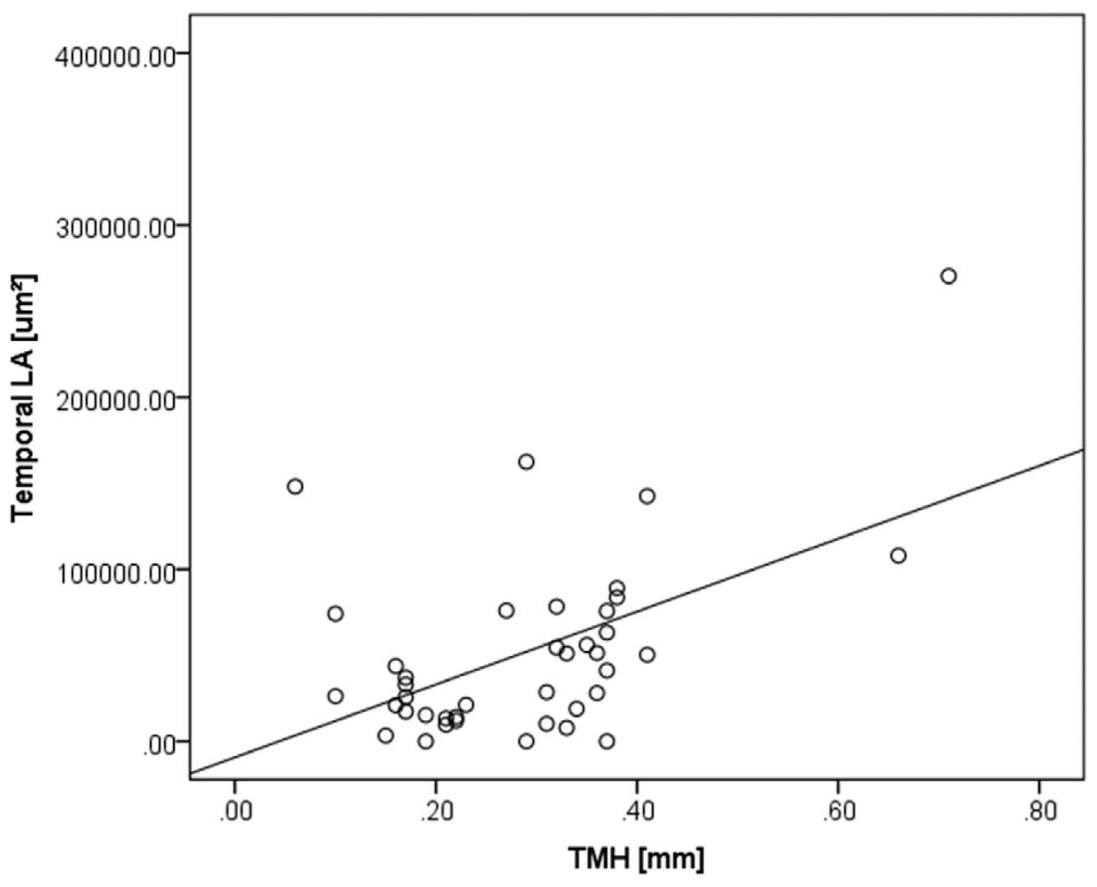

Figure 3. Correlation between central, inferior TMH and temporal LA.

$0.789, P<0.001)$, and nasal LIPCOF area was significantly correlated to nasal tear meniscus fluorescence $(r=0.415, P=$ 0.008).

Lid-parallel conjunctival fold scores were significantly correlated with tear meniscus fluorescence scores (Spearman rank: temporal, $r=0.837, P<0001$; nasal, $r=0.609, P<$ 0.001). Subjective LIPCOF grading was significantly correlated with LIPCOF area (Spearman rank: temporal, $r=0.707, P<$ 0.001 ; nasal, $r=0.515, P=0.001$ ).

A correction factor for tear meniscus height in the presence of LIPCOF was calculated, applying linear regression analyses. This was based on the sum of nasal and temporal LIPCOF scores.

\section{Linear Regression Analyses}

Scheimpflug Camera analyses of LA:

$\mathrm{TMH}_{\text {central-inferior }}=0.227+0.000000759 * \mathrm{LA} \mathrm{Sum}+\varepsilon$

Subjective LIPCOF grading:

$$
\mathrm{TMH}_{\text {central-inferior }}=0.195+0.0420 * \mathrm{LIPCOF} \text { Sum }+\varepsilon
$$

In order to adjust dry eye cutoff values for tear meniscus height, the corrected tear meniscus height cutoff values were calculated for each LIPCOF sum degree (Table 3). To assist clinicians in tear meniscus height evaluation, this was done for the subjective LIPCOF scores using Equation 3, which was based on Equation 2.

Different cutoff values of the tear meniscus are reported in the literature. Since a cutoff value of $0.20 \mathrm{~mm}$ is often used in clinical practice, this value was used as an example of the cutoff value increase in LIPCOF Sum in Table 3. However, care should be taken with this approach because of the moderate (although, statistically significant) correlations between measured tear meniscus height and LIPCOF scores (Figs. 3-5).

$$
\begin{aligned}
\mathrm{TMH}_{\text {Corrected, central-inferior }=} & \mathrm{TMH}_{\text {central-inferior }} \\
& -0.0420 * \text { LIPCOF Sum } \\
= & 0,195+\backslash \text { epsilon }
\end{aligned}
$$

\section{Discussion}

This study aimed to observe if LIPCOF had an impact on the height of the central tear meniscus, as has already been shown for paracentral tear menisci. ${ }^{21}$ Scores of LIPCOF were analyzed using a modified Scheimpflug camera. This system was shown to work well in previous research ${ }^{7,25}$ and was chosen as a more objective alternative to subjective grading of LIPCOF. The good correlation between subjective LIPCOF grading and the modified Scheimpflug camera measurements may confirm its usefulness. Furthermore, this system enabled analysis of the cross-sectional area of LIPCOF, named LIPCOF area. Other researchers have used ocular coherence tomography ${ }^{10,19,26-28}$ for the evaluation of LIPCOF; however, it appears that the modified Scheimpflug camera may serve as an alternative technique for the evaluation of LIPCOF. ${ }^{7}$

Temporal LIPCOF appeared to have a significant impact on central tear meniscus height of both the lower and upper lids, while nasal LIPCOF only seemed to impact on the lower lid tear meniscus height. Patients with increased LIPCOF degrees showed increased central tear meniscus height values. A similar effect was probably reported by Gumus et al., ${ }^{19}$ who reported an increasing severity of conjunctivochalasis with aging, as well as a significant positive correlation between tear meniscus height and age, but this is not explicitly stated. This effect may be explained by compensatory tear film production, as subjects with increased LIPCOF scores are likely to suffer from dry eye symptoms. This hypothesis may be supported by the general increase of the superior and inferior tear meniscus height in LIPCOF. However, this would mean that the majority of non-aqueous-deficient dry eye patients would have to show 


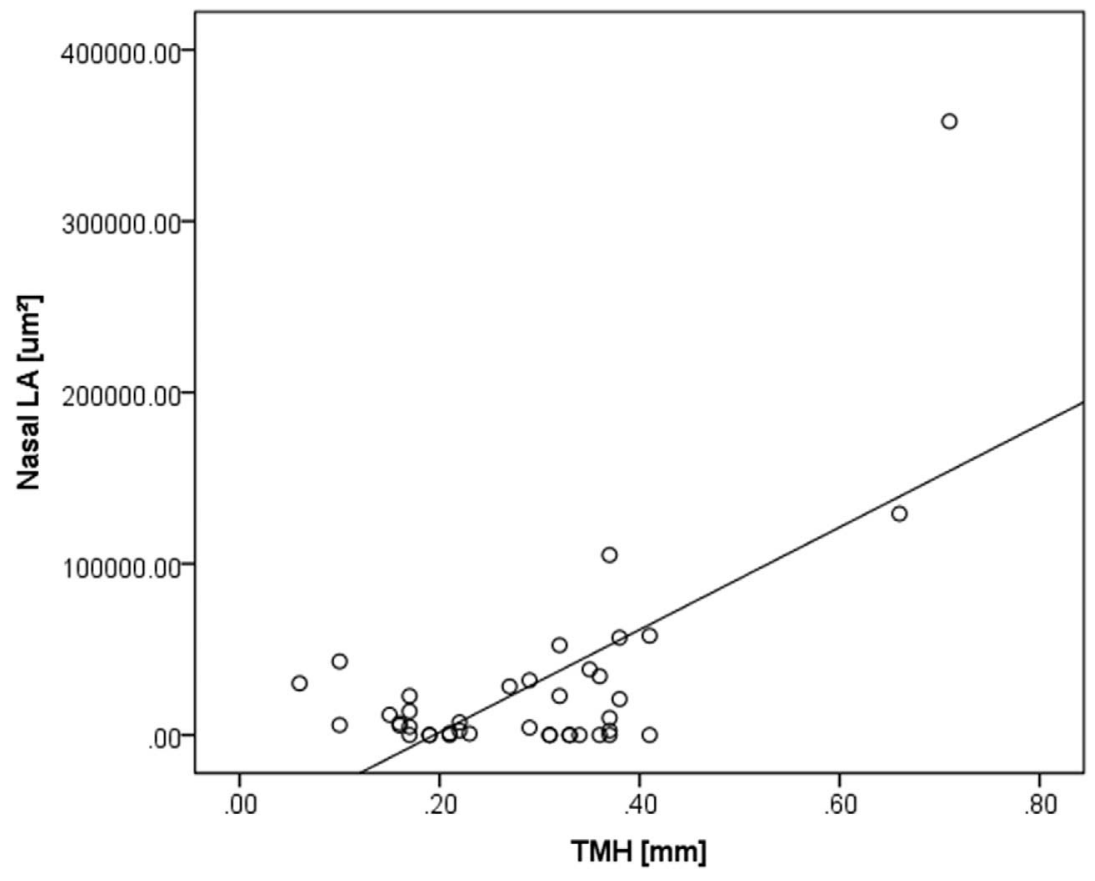

Figure 4. Correlation between inferior, central tear meniscus height (TMH) and nasal LA.

a higher tear meniscus height than healthy subjects, which is generally not the case.

A more likely mechanism for the positive correlation between LIPCOF and central tear meniscus height may represent a displacement of the tear film by the volume of LIPCOF combined with a reduced tear flow toward the tear punctum. Putting LIPCOF area into relation to the mean crosssectional area of the central tear meniscus-reported to be $15,927 \pm 4431 \mu \mathrm{m}^{2}$ (SD) in healthy and $9492 \pm 3010 \mu \mathrm{m}^{2}$ in dry eye patients ${ }^{29}$ - may demonstrate the substantial effect of LIPCOF on the tear meniscus. However, the cross-sectional area of LIPCOF appeared to be much smaller than that of conjunctivochalasis. Gumus et al. ${ }^{19}$ reported a decrease of a cross-sectional area of conjunctivochalasis from $0.247 \pm 0.24$ $\mathrm{mm}^{2}$ (SD) to $0.054 \pm 0.79 \mathrm{~mm}^{2}$ after cauterization. Even though this dramatically reduces the cross-sectional area, it shows that treated conjunctivochalasis is still commonly much larger than LIPCOF and that, even after conjunctivochalasis treatment, the remaining conjunctivochalasis may continue to have a similar effect as higher LIPCOF grades.

As reported by Veres et al., ${ }^{26}$ LIPCOF are covered by a thin tear film. This was also observed in this study. The tear meniscus fluorescence test showed an intensive fluorescence of the central tear meniscus in the presence of an increased LIPCOF score; however, there was poor or no fluorescence of

TABLE 3. Corrected Cutoff Values of the Central, Inferior TMH Based on the LIPCOF Sum Correction Factor

\begin{tabular}{lc}
\hline LIPCOF Sum & Corrected Dry Eye Cutoff Values of TMH, $\mathbf{~ m m}$ \\
\hline 0 & 0.20 \\
1 & 0.24 \\
2 & 0.28 \\
3 & 0.33 \\
4 & 0.37 \\
5 & 0.41 \\
6 & 0.45 \\
\hline
\end{tabular}

the paracentral tear menisci, precisely in the area where the LIPCOF were observed. In contrast, there was an even fluorescence visible over the complete tear meniscus area in subjects with no LIPCOF. Slit lamp observation of the tear meniscus using the Tearscope (before the tear meniscus fluorescence test) showed that the LIPCOF area was covered by tear film (Fig. 6). However, the fluorescence pattern of the tear meniscus fluorescence test demonstrated that this tear film layer was too thin to show visible fluorescence at the area where LIPCOF were observed, and that the meniscus therefore appeared to be less high at this location than at the central tear meniscus. This may strengthen the hypothesis that LIPCOF fill up the paracentral tear menisci and displace the tear meniscus toward the central portion.

As investigated by Maki et al., ${ }^{30,31}$ the lacrimal gland influx splits, with some tear fluid traveling through the upper meniscus and some traveling around the temporal canthus and into the lower meniscus. Immediately after the blink, some tear fluid travels toward the nasal canthus, and some travels toward the temporal canthus, under the effect of the two main capillary forces in the tear meniscus: one force pulling toward the tear punctum and one toward the cul-desac. However, after 10 seconds the tear flow in the upper and lower tear menisci points toward the respective tear punctum.

It can be speculated that this balance is different in the presence of LIPCOF. The nasal LIPCOF may delay the tear flux toward the tear punctum, while the temporal LIPCOF may induce a capillary force from the central portion of the tear meniscus toward the temporal folds. Such temporal LIPCOF may bind some tear film volume. Neglecting the volume of the folds behind the temporal tear meniscus, a negative correlation between temporal LIPCOF and central tear meniscus height could be reasonably due to this capillary effect. Hence, the positive correlation observed between temporal LIPCOF and the central tear meniscus height may strengthen the volumebased displacement of the tear meniscus by the temporal LIPCOF. This was confirmed by the positive correlations between LIPCOF and the upper lid tear meniscus height, 


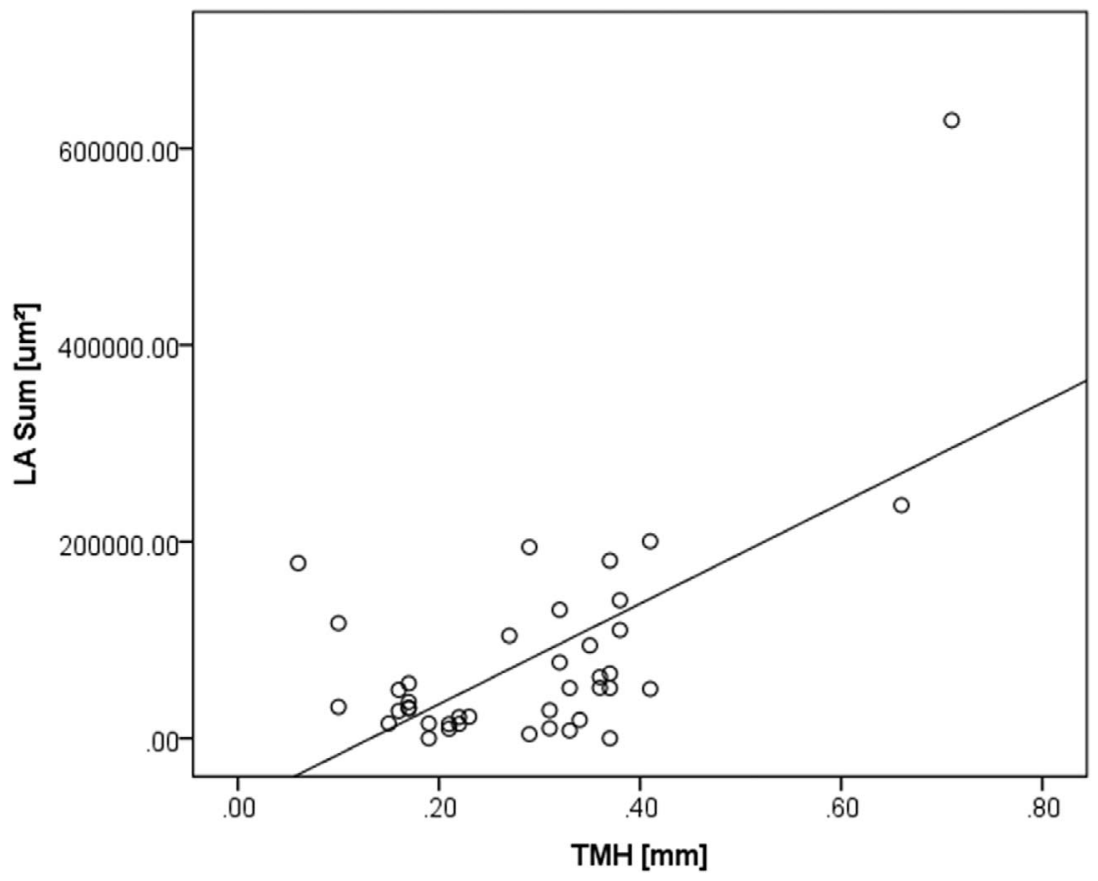

Figure 5. Correlation between inferior, central TMH and LA Sum.

while there was no significant correlation between the upper lid tear meniscus height and nasal LIPCOF. In contrast, nasal LIPCOF may mainly lead to a delayed flux toward the tear punctum. ${ }^{21}$

Nevertheless, the measurement of the central tear meniscus height appears to be an important observation in dry eye management. ${ }^{32,33}$ This study evaluated a positive relation between LIPCOF scores and central tear meniscus height, indicating a possible misinterpretation of the central tear meniscus height. This calls into question the reliability of tear meniscus height measurements. This may be important information for those using tear meniscus height as part of a dry eye assessment, both in the clinical and research setting. It can be suggested that common cutoff values of the tear meniscus height need to be corrected in those patients presenting with increased LIPCOF scores

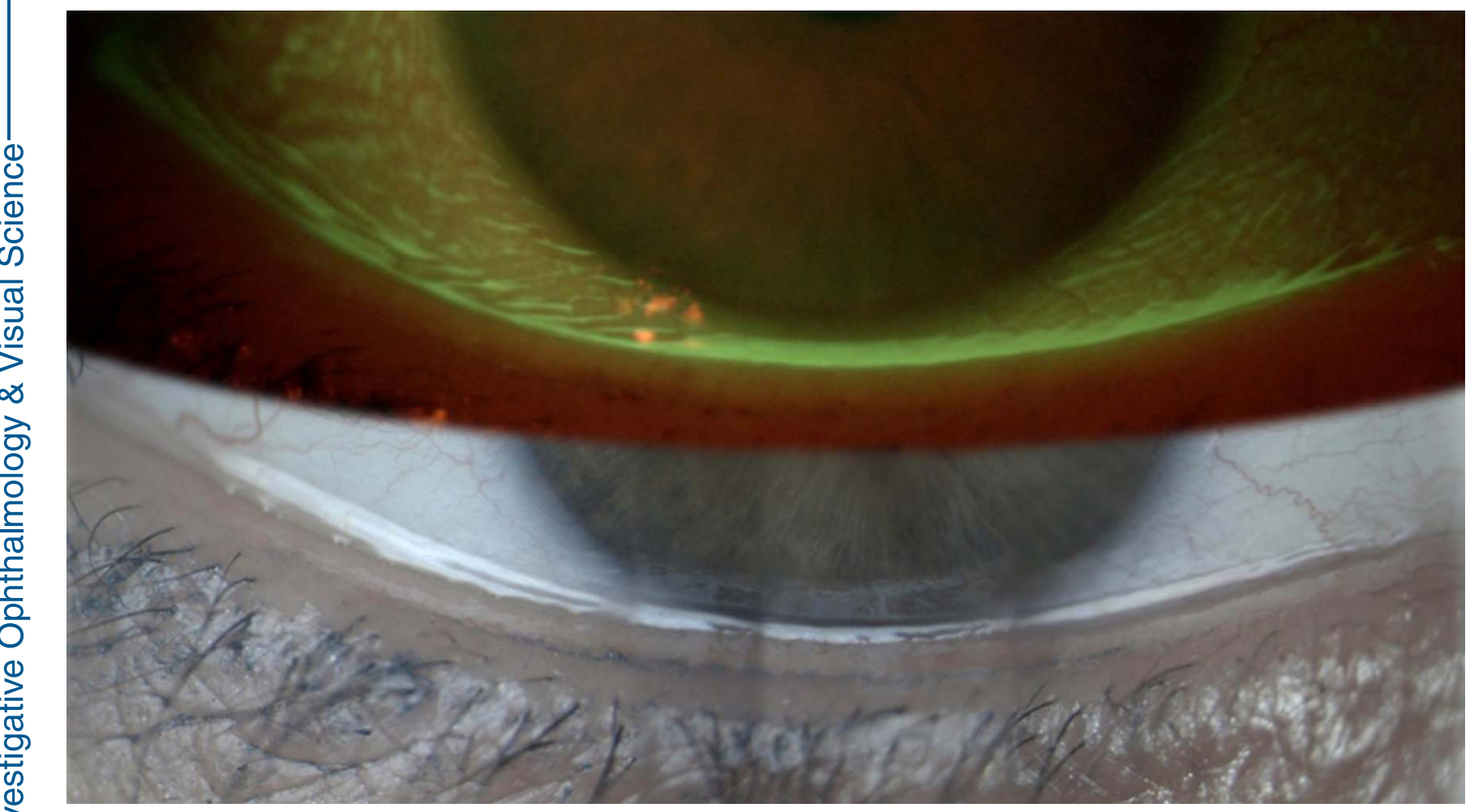

Figure 6. Observation of the tear meniscus first by using the Tearscope, followed by the TMF test. 
(Table 3). Furthermore, in patients with increased LIPCOF scores, alternative methods to evaluate tear film volume may need to be considered in clinical practice and research. Since the effect of LIPCOF on the superior tear meniscus was less strong than on the inferior tear meniscus, superior tear meniscus observation may be an alternative or confirming method of inferior tear meniscus measurements. Other alternatives may, for example, be the use of the phenol red thread test, ${ }^{34,35}$ the evaluation of the tear meniscus volume applying fluophotometry, ${ }^{36}$ or observing the tear meniscus dimensions along the full eye lid using ocular coherence tomography. ${ }^{37}$

\section{Conclusions}

The correlations between LIPCOF/LIPCOF area scores and tear meniscus fluorescence may indicate that the tear meniscus fluorescence test has a potential to assist LIPCOF evaluation. In the presence of increased LIPCOF, the main portion of the paracentral tear menisci consists of LIPCOF, however, and not tear film. The volume of the tear meniscus in healthy patients may be correctly evaluated by central tear meniscus height measurement. However, in patients with LIPCOF, the real tear film volume may be smaller than the tear meniscus height measurement may suggest.

\section{Acknowledgments}

We thank Oculus Optikgeräte $\mathrm{GmbH}$, Wetzlar, Germany, and Andreas Steinmüller, R\&D, Oculus Oculus Optikgeräte GmbH, for providing the Pentacam HD, including the modified software to our specifications.

The authors alone are responsible for the content and writing of the paper.

Disclosure: H. Pult, None; B.H. Riede-Pult, None

\section{References}

1. Murube J. Characteristics and etiology of conjunctivochalasis: historical perspective. Ocul Surf. 2005;3:7-14.

2. Hughes W. Conjunctivochalasis. Am J Ophthalmol. 1942;25: 48-51.

3. Höh H, Schirra F, Kienecker C, Ruprecht KW. Lid-parallel conjunctival folds are a sure diagnostic sign of dry eye. Ophthalmologe. 1995;92:802-808.

4. Pult H, Purslow C, Murphy PJ. The relationship between clinical signs and dry eye symptoms. Eye (Lond). 2011;25: 502-510.

5. Berry M, Pult H, Purslow C, Murphy PJ. Mucins and ocular signs in symptomatic and asymptomatic contact lens wear. Optom Vis Sci. 2008;85:E930-E938.

6. Pult H, Purslow C, Berry M, Murphy PJ. Clinical tests for successful contact lens wear: relationship and predictive potential. Optom Vis Sci. 2008;85:E924-E929.

7. Pult H, Riede-Pult BH. Analyses of lid parallel conjunctival folds using a Scheimpflug camera. Paper presented at: British Contact Lens Association conference; June 6-9, 2014; Birmingham, UK.

8. Pult H, Murphy PJ, Purslow C. A novel method to predict the dry eye symptoms in new contact lens wearers. Optom Vis Sci. 2009;86:E1042-E1050.

9. Pult H, Purslow C, Murphy PJ, Berry M. Lid wiper epitheliopathy, ocular surface and tear film in symptomatic contact lens wearers. Acta Ophthalmologica. 2008;86:0. doi:10.1111/j. 1755-3768.2008.4334.x
10. Németh J, Fodor E, Lang Z, et al. Lid-parallel conjunctival folds (LIPCOF) and dry eye: A multicentre study. Br J Ophthalmol. 2012;96:1380-1385.

11. Dausch D, Lee S, Dausch S, Kim JC, Schwert G, Michelson W. Comparative study of treatment of the dry eye syndrome due to disturbances of the tear film lipid layer with lipid-containing tear substitutes. Klin Monatsbl Augenheilkd. 2006;223:974983.

12. Pult H, Riede-Pult B. Impact of lid-parallel conjunctival folds on the tear meniscus thickness. Paper presented at: British Contact Lens Association conference; June 6-9, 2014; Birmingham, UK.

13. Gaffney EA, Tiffany JM, Yokoi N, Bron AJ. A mass and solute balance model for tear volume and osmolarity in the normal and the dry eye. Prog Retin Eye Res. 2010;29:59-78.

14. Holly FJ. Physical chemistry of the normal and disordered tear film. Trans Ophthalmol Soc U K. 1985;104(Pt 4);374-380.

15. Wang J, Aquavella J, Palakuru J, Chung S, Feng C. Relationships between central tear film thickness and tear menisci of the upper and lower eyelids. Invest Ophthalmol Vis Sci. 2006;47: 4349-4355.

16. Uchida A, Uchino M, Goto E, et al. Noninvasive interference tear meniscometry in dry eye patients with Sjogren syndrome. Am J Ophthalmol. 2007; 144:232-237.

17. Savini G, Barboni P, Zanini M. Tear meniscus evaluation by optical coherence tomography. Ophthalmic Surg Lasers Imaging. 2006;37:112-118.

18. Yokoi N, Bron AJ, Tiffany JM, Maruyama K, Komuro A, Kinoshita S. Relationship between tear volume and tear meniscus curvature. Arch Ophthalmol. 2004;122:1265-1269.

19. Gumus K, Pflugfelder SC. Increasing prevalence and severity of conjunctivochalasis with aging detected by anterior segment optical coherence tomography. Am J Ophthalmol. 2013;155:238-242.e232.

20. Wang Y, Dogru M, Matsumoto Y, et al. The impact of nasal conjunctivochalasis on tear functions and ocular surface findings. Am J Ophthalmol. 2007;144:930-937.e931.

21. Bandlitz S, Purslow C, Murphy PJ, Pult H. The relationship between tear meniscus regularity and conjunctival folds. Optom Vis Sci. 2014;91:1037-1044.

22. Pult H, Murphy PJ, Purslow CA. Novel method to predict dry eye symptoms in new contact lens wearers. Optom Vis Sci. 2009;86:E1042-E1050.

23. Graham AD, Truong TN, Lin MC. Conjunctival epithelial flap in continuous contact lens wear. Optom Vis Sci. 2009;86:e324331.

24. Pult H, Riede-Pult BA. New modified fluorescein strip: its repeatability and usefulness in tear film break-up time analysis. Cont Lens Anterior Eye. 2012;35:35-38.

25. Pult H, Murphy P, Riede-Pult BH. Velocity of upper lid in spontaneous complete blinks and dry eye. Paper presented at: British Contact Lens Association conference; June 6-9, 2014; Birmingham, UK.

26. Veres A, Tapaszto B, Kosina-Hagyo K, Somfai GM, Nemeth J. Imaging lid-parallel conjunctival folds with OCT and comparing its grading with the slit lamp classification in dry eye patients and normal subjects. Invest Ophthalmol Vis Sci. 2011;52:2945-2951.

27. Tapasztó B, Veres A, Kosina-Hagyó K, Somfai GM, Németh J. OCT imaging of lid-parallel conjunctival folds in soft contact lens wearers. Optom Vis Sci. 2011;88:1206-1213.

28. Gumus K, Crockett CH, Pflugfelder SC. Anterior segment optical coherence tomography: a diagnostic instrument for conjunctivochalasis. Am J Ophthalmol. 2010;150:798-806.

29. Shen M, Li J, Wang J, et al. Upper and lower tear menisci in the diagnosis of dry eye. Invest Ophthalmol Vis Sci. 2009;50: 2722-2726. 
30. Maki KL, Braun RJ, Henshaw WD, King-Smith PE. Tear film dynamics on an eye-shaped domain I: pressure boundary conditions. Math Med Biol. 2010;27:227-254.

31. Maki KL, Braun RJ, Ucciferro P, Henshaw WD, King-Smith PE. Tear film dynamics on an eye-shaped domain. Part 2. Flux boundary conditions. J Fluid Mech. 2010;647:361-390.

32. Lemp MA, Baudouin $\mathrm{C}$, Baum $\mathrm{J}$, et al. The definition and classification of dry eye disease: Report of the Definition and Classification Subcommittee of the International Dry Eye WorkShop (2007). Ocul Surf. 2007;5:75-92.

33. Bron AJ, Bron AJ, Abelson MB, et al. Methodologies to diagnose and monitor dry eye disease: Report of the Diagnostic Methodology Subcommittee of the International Dry Eye WorkShop (2007). Ocul Surf. 2007;5:108-152.
34. Miller WL, Doughty MJ, Narayanan S, et al. A comparison of tear volume (by tear meniscus height and phenol red thread test) and tear fluid osmolality measures in non-lens wearers and in contact lens wearers. Eye Contact Lens. 2004;30:132137.

35. Kurihashi K, Yanagihara N, Honda Y. A modified Schirmer test: the fine-thread method for measuring lacrimation. J Pediatr Ophthalmol. 1977;14:390-397.

36. Eter N, Gobbels M. A new technique for tear film fluorophotometry. Br J Ophthalmol. 2002;86:616-619.

37. Tung CI, Perin AF, Gumus K, Pflugfelder SC. Tear meniscus dimensions in tear dysfunction and their correlation with clinical parameters. Am J Ophthalmol. 2014;157:301310.e301. 